\title{
A Chinese Herbal Medicine, Tokishakuyakusan, Reduces the Worsening of Impairments and Independence after Stroke: A 1-Year Randomized, Controlled Trial
}

\author{
Hirozo Goto, ${ }^{1}$ Nobuhiko Satoh, ${ }^{2}$ Yoshinori Hayashi, ${ }^{3}$ Hiroaki Hikiami, ${ }^{1}$ \\ Yutaka Nagata, ${ }^{1}$ Ryosuke Obi, ${ }^{1}$ and Yutaka Shimada ${ }^{1}$ \\ ${ }^{1}$ Department of Japanese Oriental Medicine, Graduate School of Medicine and Pharmaceutical Sciences, University of Toyama, \\ Toyama 930-0194, Japan \\ ${ }^{2}$ Tonami General Hospital, Toyama, Japan \\ ${ }^{3}$ Yoshimi Hospital, Toyama, Japan \\ Correspondence should be addressed to Hirozo Goto, hiro510@med.u-toyama.ac.jp
}

Received 9 August 2008; Accepted 3 March 2009

Copyright (๑) 2011 Hirozo Goto et al. This is an open access article distributed under the Creative Commons Attribution License, which permits unrestricted use, distribution, and reproduction in any medium, provided the original work is properly cited.

In post-stroke patients, the recurrence of stroke and progression of impairments lead to a bedridden state and dementia. As for their treatments, only anti-hypertension and anti-coagulation therapies to prevent the recurrence of stroke are available. In Asia, post-stroke patients with impairments are often treated with herbal medicine. The present study evaluated the effectiveness of tokishakuyakusan (TS) in improving the impairment and independence in post-stroke patients. Thirty-one post-stroke patients (mean age $=81.4$ years) were recruited and enrolled. Participants were randomly assigned to the TS group $(n=16)$ or nontreatment (control) group $(n=15)$ and treated for 12 months. Impairments were assessed using the Stroke Impairment Assessment Set (SIAS). Independence was evaluated using the functional independence measure (FIM). For each outcome measure, mean change was calculated every 3 months. The results were that impairments according to SIAS did not significantly change in the TS group. In contrast, SIAS significantly worsened in the control group. There was a significant difference between the two groups. In each term of SIAS, affected lower extremity scores, abdominal muscle strength, function of visuospatial perception, and so forth. in the TS group were better than those in the control group. Independence according to FIM did not change significantly in the TS group. In contrast, FIM significantly worsened in the control group. There was also a significant difference between the two groups. In conclusion, TS was considered to suppress the impairments of lower limbs and to exert a favorable effect on cerebral function for post-stroke patients.

\section{Introduction}

In post-stroke patients, the recurrence of stroke and progression of impairments lead to a bedridden state and dementia. These are important medical problems in many aging societies. However, as for their treatments, only antihypertension and anti-coagulation therapies to prevent the recurrence of stroke are available. In terms of non-drug therapy, only rehabilitation is used for inhibiting the progression of impairments $[1,2]$.

In East Asia, post-stroke patients with impairments are often treated with herbal medicine [3], acupuncture and moxibustion [4, 5]. Tokishakuyakusan (TS), a traditional Chinese herbal medicine called Dang-gui-shao-yao-san in Chinese, is reported to have a neuroendocrine effect [6] and to activate choline acetyltransferase [7]. Recently, the efficacy of TS on cognitive impairment from Alzheimer's disease [8] and improvement of microcirculation in patients with asymptomatic cerebral infarction [9] have been reported clinically. A neuroprotective effect and the improvement of microcirculation are thought to be useful for post-stroke patients. But there has been no report on the long-term effect of TS for post-stroke patients.

The aim of this study was to determine whether TS would improve the impairments and independence of post-stroke patients over a 12 -month period in a randomized, controlled trial.

\section{Methods}

2.1. Participants. Subjects were recruited from two longterm-care facilities located in Toyama prefecture, Japan. 
The diagnosis of post-stroke was made on the basis of a history of cerebral bleeding, infarction or subarachnoid hemorrhage as well as having paralysis due to cerebral lesion. The patients had already passed the acute phase of stroke and had been stable for the previous year. At baseline, each patient underwent a uniform evaluation that included medical history, physical and neurological examination, as well as assessment of impairments and independence.

Impairments were assessed using the Stroke Impairment Assessment Set (SIAS), a standardized measure of stroke impairment consisting of the subcategories of motor, tone, sensory, range of motion, pain, trunk function, visuospatial function, speech and unaffected side function, with its high inter-rater reliability having been reported $[10,11]$. Independence status was assessed by Functional Independence Measure (FIM), a modality now being used throughout the world [12]. Patients with severe dementia, were bedridden, or had neoplastic or other disease that would likely prevent completion of this study, were excluded. As a result, 31 patients ( 9 men and 22 women: mean age $\pm \mathrm{SD}, 81.4 \pm 8.2$ years) were selected. Mean post-stroke duration of all subjects was $68.3 \pm 75.3$ months. As for original disease, 23 had cerebral infarction, seven had cerebral bleeding and one had subarachnoid hemorrhage. Their complications mainly consisted of hypertension, diabetes and ischemic heart disease, but they were well controlled in this study period. Twelve of the subjects had been using anti-coagulation drugs prior to initiation of this study. All subjects were receiving physio- and ergotherapy.

The study design was approved by the Human Subjects Committee, University of Toyama. All patients provided written informed consent in accordance with ethical guidelines set forth in the 1975 Declaration of Helsinki.

2.2. Intervention Protocol. A 12-month randomized, controlled trial was begun between October 2005 and January 2006. Participants were assigned to the TS and control groups using a table of random numbers (Table 1).

TS, which is approved for medical use in Japan, was purchased from Tsumura Co. Ltd (Tokyo, Japan). It consists of six herbs: $4.0 \mathrm{~g}$ of Alismatis Rhizoma (Alisma orientale JUZEPCZUK), $4.0 \mathrm{~g}$ of Paeoniae Radix (Paeonia lactiflora PALLAS), $4.0 \mathrm{~g}$ of Atractylodis Rhizoma (Atractylodes lancea DE CANDOLLE), $4.0 \mathrm{~g}$ of Hoelen (Poria cocos WOLF), $3.0 \mathrm{~g}$ of Cnidii Rhizoma (Cnidium officinale MAKINO) and $3.0 \mathrm{~g}$ of Angelicae Radix (Angelica acutiloba KITAGAWA). The aqueous extract was lyophilized to obtain powder. Lactose $(3.5 \mathrm{~g})$ was added to the powder $(4.0 \mathrm{~g})$ to make granules (total $7.5 \mathrm{~g}$ ). Subjects were orally administered TS at $7.5 \mathrm{~g} /$ day, $2.5 \mathrm{~g}$ three times daily, $30 \mathrm{~min}$ after meals for 12 months.

2.3. Outcome Determination. The SIAS scores were measured by physiotherapists who did not know which subjects were being treated with TS. The FIM scores were measured by care workers who had no knowledge of who the TStreated subjects were. Every score was determined five times: at baseline, and every 3 months up to 12 months, the
TABLE 1: Clinical and demographic characteristics of study participants.

\begin{tabular}{lcc}
\hline Group & TS group & Control group \\
\hline Sex (male/female) & $4 / 12$ & $5 / 10$ \\
Age, mean \pm SD & $81.0 \pm 9.4$ & $81.9 \pm 7.1$ \\
$\begin{array}{l}\text { Duration of illness, months, } \\
\text { mean } \pm \text { SD }\end{array}$ & $77.3 \pm 86.9$ & $58.6 \pm 62.2$ \\
Disease & & \\
$\quad$ Cerebral infarction & 11 & 12 \\
$\quad$ Cerebral bleeding & 4 & 3 \\
$\quad$ Subarachnoidal hemorrhage & 1 & 0 \\
Complication & 11 & 9 \\
$\quad$ Hypertension & 4 & 5 \\
$\quad$ Diabetes mellitus & 1 & 3 \\
$\quad$ Ischemic heart disease & 5 & 7 \\
Anti-coagulant drugs &
\end{tabular}

Note: All group comparisons $P>.05$. SD: standard deviation; TS: Tokishakuyakusan.

completion of the protocol. Body weights were measured at the same time points.

2.4. Statistical Analysis. Changes in SIAS, FIM and body weight from baseline (mean $\pm \mathrm{SD}$ ) were compared by post hoc test with repeated measures of analysis of variance (ANOVA). Other data from baseline to endpoint were also compared by Wilcoxon signed-rank test with Bonferroni correction. $P<.05$ was required for statistical significance.

\section{Results}

3.1. Characteristics of Participants. Only one patient in the TS group withdrew at 6 weeks into the trial because of numbness in his limbs; besides TS, he had been taking amantadine hydrochloride and captopril from a few weeks prior to the start of the trial. As a result, 30 patients completed the protocol. Participants were predominantly female. There were no significant differences in baseline characteristics between the TS and control groups in terms of age, sex, poststroke interval, original disease or complications. Subjects had a mean SIAS score of $44.3 \pm 18.1$, a mean FIM score of $64.6 \pm 25.6$, and a mean body weight of $43.3 \pm 7.5 \mathrm{~kg}$. There were no significant differences in baseline scores and body weights between the TS and control groups (Table 2).

3.2. Changes in SIAS and Each Item. The mean SIAS score in the TS group did not change through the 12-month trial duration, whereas that in the control group worsened significantly $(P<.05)$, the difference between the two groups being significant $(P<.05)$. At any of the 3 -month terms of SIAS, the next scores were significantly different between the two groups. The affected lower extremity scores, which were the knee-extension test and the foot-pat test, did not change from the beginning to 12 months in the TS group, but they decreased significantly in the control group 
TABLE 2: Changes in SIAS, FIM and body weight of TS and control groups.

\begin{tabular}{|c|c|c|c|c|c|c|c|c|}
\hline Outcome & Range & Group & Baseline & 3 months & 6 months & 9 months & 12 months & $P$-value ${ }^{\mathrm{a}}$ \\
\hline \multirow{2}{*}{ SIAS } & \multirow{2}{*}{$0-76$} & TS & $43.6 \pm 20.6$ & $45.0 \pm 21.5$ & $43.6 \pm 20.6$ & $43.6 \pm 20.7$ & $43.4 \pm 20.5$ & \multirow{2}{*}{.044} \\
\hline & & Control & $43.6 \pm 15.9$ & $43.3 \pm 15.6$ & $41.9 \pm 14.9$ & $41.2 \pm 14.7$ & $36.9 \pm 16.6^{*}$ & \\
\hline \multirow{2}{*}{ FIM } & \multirow{2}{*}{$0-126$} & TS & $66.1 \pm 29.3$ & $65.5 \pm 31.0$ & $66.3 \pm 30.6$ & $65.4 \pm 31.3$ & $66.2 \pm 31.5$ & \multirow{2}{*}{.008} \\
\hline & & Control & $60.8 \pm 21.4$ & $58.3 \pm 21.4$ & $56.7 \pm 20.9$ & $55.8 \pm 20.8$ & $49.2 \pm 20.7^{*}$ & \\
\hline \multirow{2}{*}{ Body weight } & \multirow{2}{*}{$(\mathrm{kg})$} & TS & $41.6 \pm 7.1$ & $41.7 \pm 7.8$ & $41.8 \pm 7.4$ & $40.5 \pm 7.3$ & $40.1 \pm 7.4^{*}$ & \multirow{2}{*}{.64} \\
\hline & & Control & $44.6 \pm 7.8$ & $44.3 \pm 7.9$ & $43.9 \pm 7.6$ & $42.6 \pm 7.7$ & $41.9 \pm 7.4^{* *}$ & \\
\hline
\end{tabular}

a Comparison of 12-month changes between TS group and control group.

${ }^{*} P<.05,{ }^{*} P<.01$, comparison from baseline to 12 months in the same group.

TABLE 3: Comparison between TS and control groups on each item of SIAS.

\begin{tabular}{|c|c|c|c|c|c|c|}
\hline \multirow{2}{*}{ Variable } & \multirow{2}{*}{ Range } & \multicolumn{2}{|c|}{ TS group } & \multicolumn{2}{|c|}{ Control group } & \multirow{2}{*}{$P$-value ${ }^{\mathrm{a}}$} \\
\hline & & Baseline & 12 months & Baseline & 12 months & \\
\hline Knee-mouse & $0-5$ & $2.53 \pm 1.92$ & $2.40 \pm 0.51$ & $2.13 \pm 1.77$ & $1.73 \pm 1.75^{*}$ & .295 \\
\hline Finger-function & $0-5$ & $2.33 \pm 1.76$ & $2.27 \pm 1.87$ & $1.87 \pm 1.55$ & $1.40 \pm 1.35^{*}$ & .197 \\
\hline Hip-function & $0-5$ & $2.40 \pm 1.50$ & $2.27 \pm 1.58$ & $2.33 \pm 1.40$ & $1.80 \pm 1.37^{* *}$ & .262 \\
\hline Knee-extension & $0-5$ & $2.27 \pm 1.44$ & $2.27 \pm 1.39$ & $2.13 \pm 1.46$ & $1.40 \pm 1.12^{* *}$ & $<.001$ \\
\hline Foot-pat & $0-5$ & $2.33 \pm 1.45$ & $2.27 \pm 1.39$ & $2.27 \pm 1.67$ & $1.73 \pm 1.44^{* *}$ & .018 \\
\hline DTR & $0-3$ & $1.83 \pm 0.98$ & $1.90 \pm 0.87$ & $2.10 \pm 0.81$ & $2.00 \pm 0.78$ & .106 \\
\hline Tone & $0-3$ & $2.10 \pm 1.04$ & $1.83 \pm 1.10$ & $2.10 \pm 0.81$ & $2.00 \pm 0.80$ & .670 \\
\hline Touch & $0-3$ & $2.30 \pm 0.75$ & $2.13 \pm 0.74$ & $2.17 \pm 0.77$ & $1.83 \pm 0.92$ & .237 \\
\hline Position & $0-3$ & $1.87 \pm 1.06$ & $1.87 \pm 1.13$ & $1.97 \pm 0.90$ & $1.77 \pm 0.98$ & .219 \\
\hline Range of motion & $0-3$ & $1.87 \pm 1.06$ & $1.87 \pm 1.13$ & $1.97 \pm 0.90$ & $1.76 \pm 0.98$ & .219 \\
\hline Pain & $0-3$ & $2.53 \pm 0.64$ & $2.47 \pm 0.64$ & $2.73 \pm 0.46$ & $2.73 \pm 0.46$ & .560 \\
\hline Abdominal MMT & $0-3$ & $1.40 \pm 1.06$ & $1.67 \pm 1.18^{*}$ & $1.33 \pm 0.82$ & $1.20 \pm 0.86$ & .004 \\
\hline Verticality & $0-3$ & $2.27 \pm 0.80$ & $2.13 \pm 0.99$ & $2.07 \pm 0.59$ & $1.73 \pm 0.88^{*}$ & .359 \\
\hline Visuo-spat. score & $0-3$ & $1.67 \pm 1.35$ & $1.80 \pm 1.42$ & $1.67 \pm 0.98$ & $1.33 \pm 1.18^{*}$ & .031 \\
\hline Speech & $0-3$ & $2.00 \pm 0.93$ & $1.87 \pm 0.92$ & $1.73 \pm 0.70$ & $1.40 \pm 0.74$ & .681 \\
\hline Unaffected quadriceps & $0-3$ & $1.80 \pm 0.78$ & $1.87 \pm 0.74$ & $1.93 \pm 0.70$ & $1.53 \pm 0.83^{*}$ & .022 \\
\hline Unaffected grip power (kg) & & $8.93 \pm 6.20$ & $8.07 \pm 6.31$ & $10.7 \pm 7.16$ & $8.80 \pm 7.67$ & .671 \\
\hline
\end{tabular}

DTR, deep tender reflexes; MMT, manual muscle testing; Visuo-spat., visuospatial perception.

${ }^{a}$ Comparison of 12 -month changes between TS group and control group.

${ }^{*} P<.05,{ }^{*} P<.01$, Comparison between baseline and at 12 months in the same group.

$(P<.01)$. Abdominal muscle strength, a measure of the function of the trunk of the body, improved from the beginning to 12 months in the TS group $(P<.05)$ but it did not change in the control group during the 12 months. The function of visuospatial perception did not change from the beginning to 12 months in the TS group, but it decreased significantly in the control group $(P<.05)$. The muscle strength of unaffected quadriceps, important for maintaining the activity of daily living, did not change in the TS group, but it decreased significantly in the control group $(P<.05)$ (Table 3).

3.3. Changes in FIM and Body Weight. The mean FIM score in the TS group did not change during the 12 months, whereas that in the control group worsened significantly $(P<.05)$, the difference between the two groups being significant $(P<.01)$. The body weights of both groups decreased significantly during the 12 months, but there were no significant differences between the two groups (Table 2). In the control group, two subjects had recurrent stroke between 9 and 12 months.

\section{Discussion}

During the long-term period of 12 months, despite the limitation of the small study population, this preliminary and randomized trial suggested that TS inhibited the worsening of impairments and independence in post-stroke patients. As for the method of stroke impairment assessment, several kinds have been developed, such as the Canadian Neurological Scale [13] and NIH Scale [14]. SIAS, used in this study, is the assessment based on the guidelines of the Buffalo Symposium of 1989 [15], and has recently been often used clinically to assess the impairment in post-stroke patients in Japan, as it can assess not only motor functions but also sensory functions and range of motion at the joints [11]. 


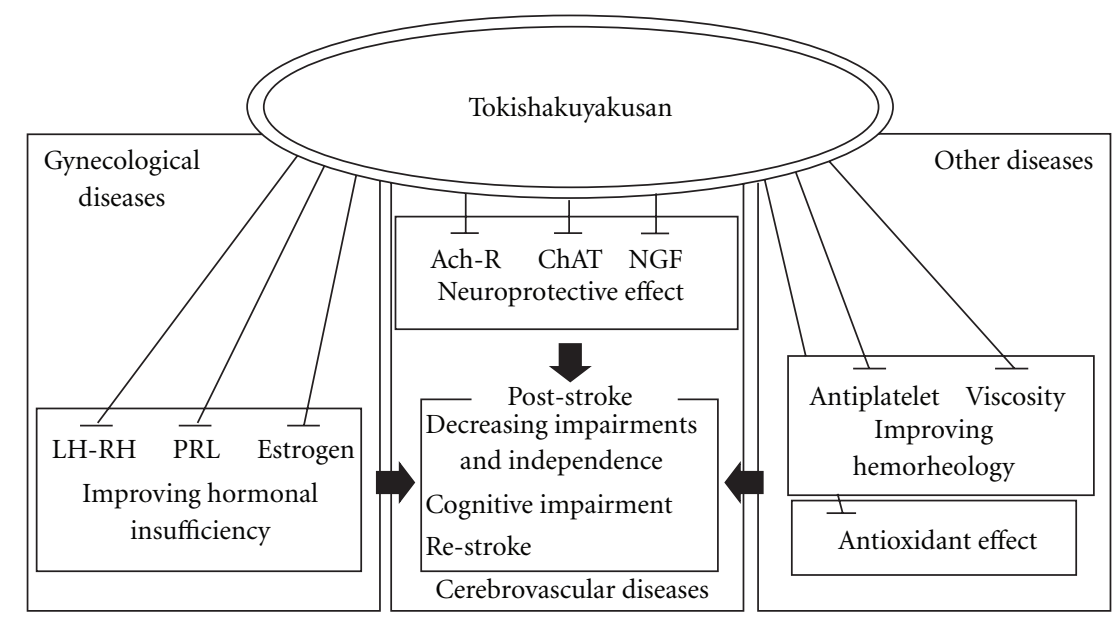

FIGURE 1: Hypothetical representation of the effects of TS on post-stroke patents. LH-RH: luteum hormone releasing hormone; PRL: prolactin; Ach-R: acetylcholine receptor; ChAT: choline acetyltransferase; NGF: nerve growth factor.

The maximum score on SIAS is 76 points, and participants with SIAS scores around 40 points do not have not complete paralysis, but they do have paresis in affected limbs. Independence was evaluated using FIM, a measurement in use worldwide [12]. The maximum score on FIM is 126 points, and participants with FIM scores around 60 points have a physical state needing slight to middle-level care. Aged participants who have had paresis for 5 or 6 years will show slowly worsening impairments and independence. With the recurrence of stroke, their condition will worsen precipitously.

Recently, by reason of being unable to live alone, many post-stroke patients, whose impairments are of mild or medium grade, are living in long-term care facilities in Japan. In this respect then, participants in this study are not rare cases, and their impairments advance step by step, finally leading to a bedridden state. As this is now a severe medical problem in Japan, any method of treatment that can improve this situation would be more than welcome. There are a few reports concerning the study of the effects of traditional Chinese medicine in post-stroke patients. The effects on aspiration pneumonia [16] and cognitive function [17, 18] of post-stroke patients for the short term were reported. Of course, maintaining function and ability, and prolonging the period of social life are also of major importance. In this regard, TS is one of the most common formulas in oriental traditional medicine and has been used in large numbers of patients for 2000 years. The administration and safety of TS have been clinically established. It is often used for gynecological diseases $[19,20]$. There are some reports on climacteric symptoms [21] and improvement of luteal insufficiency [22]. These reports are interesting, as TS has been reported to improve memory disturbance in menopausal rats [7]. In addition, TS is used for dermatological diseases [23] as well as many other medical conditions [24].

Recently there have been some reports about the treatment of patients with cognitive difficulties with Kampo medicine, as for example, the effect of kihito and BaWei Di Huang Wan on cognitive function $[25,26]$, and the effect of
Yi-Gan San on psychiatric symptoms and sleep structure in dementia patients [27]. But the mechanisms of the functions of these formulas are not yet sufficiently clear. In this study, TS was effective in suppressing impairments of lower limbs and in favorably influencing visuospatial perception. But there are few reports regarding the improvement of muscle strength and paralysis in neurological diseases treated with TS. The mechanisms of these effects are thought to be related to activation of brain function, to have a suppressive effect against re-stroke, and to ameliorate weakness in muscle strength. There are also some reports on TS and the central nervous system. The synthesis and release of neurotransmitters such as acetylcholine, dopamine and norepinephrine have been reported before $[6,7]$. Recently, in terms of neuroprotection, the protective effect against amyloid $\beta$ induced neuronal damage [28] and that after peripheral facial nerve axotomy [29] have been reported. Further, concerning blood circulation, antioxidant and antiplatelet effects [30] and regulation of genes associated with thrombosis [31] have been reported. Clinically, TS has been studied in terms of its efficacy against Alzheimer-type dementia [32], and recently its effect on mild cognitive impairment was reported [8]. Furthermore, we previously demonstrated that TS improved the microcirculation in patients with asymptomatic cerebral infarction. Its mechanisms were believed to improve the hemorheological features of viscosity and erythrocyte deformability and to decrease plasma lipid peroxides [9]. Such favorable effects, which protect neurons and improve cerebral blood circulation, would suppress the worsening of impairments and independence in poststroke patients. Furthermore, as shown by the improvement of visuospatial perception, TS affected cerebral function, especially the parietal lobe [33]. Another Kampo formula, Chotosan, was also reported to have some activity in frontal lobe function [34]. A hypothetical representation of the effects of TS on post-stroke patients is summarized in Figure 1.

In the present study, subjects were separated into two groups randomly, and they were assessed using SIAS and 
FIM to easily study impairment and independence. The measurements of SIAS and FIM were used to represent as objective an assessment as possible. The SIAS scores were measured by physiotherapists who were unaware of which subjects were being treated with TS. The FIM scores were measured by similarly blinded care workers. But without the use of placebo, the need for a blind cohort was obviously not satisfied. Although placebo is hard to use in a long-term trial such as the present 1-year term, a well-controlled trial of TS with a larger sample size and the use of comparison drugs will be needed in the future.

In every aging society, keeping the increasing populations of bed-ridden post-stroke patients to a minimum is of major importance, making the findings of this preliminary study, demonstrating that TS could slow deteriorations in impairment and independence in post-stroke subjects, particularly interesting.

\section{Funding}

Grant-in-aid for Funds for Comprehensive Research on Aging and Health from the Japanese Ministry of Health, Labor and Welfare.

\section{Acknowledgment}

The authors are grateful to Mr Arndt Gerz and Mr Thomas Fenwick for overseeing the translation into English.

\section{References}

[1] I. G. L. Van De Port, S. Wood-Dauphinee, E. Lindeman, and G. Kwakkel, "Effects of exercise training programs on walking competency after stroke: a systematic review," American Journal of Physical Medicine and Rehabilitation, vol. 86, no. 11, pp. 935-951, 2007.

[2] J. Ramas, A. Courbon, F. Roche, F. Bethoux, and P. Calmels, "Effect of training programs and exercise in adult stroke patients: literature review," Annales de Readaptation et de Medecine Physique, vol. 50, no. 6, pp. 438-444, 2007.

[3] B. Wu, M. Liu, H. Liu et al., "Meta-analysis of traditional Chinese patent medicine for ischemic stroke," Stroke, vol. 38, no. 6, pp. 1973-1979, 2007.

[4] S.-K. Moon, Y.-K. Whang, S.-U. Park et al., "Antispastic effect of electroacupuncture and moxibustion in stroke patients," American Journal of Chinese Medicine, vol. 31, no. 3, pp. 467474, 2003.

[5] D. N. Alexander, S. Cen, K. J. Sullivan, G. Bhavnani, X. Ma, and S. P. Azen, "Effects of acupuncture treatment on poststroke motor recovery and physical function: a pilot study," Neurorehabilitation and Neural Repair, vol. 18, no. 4, pp. 259-267, 2004.

[6] N. Hagino, "An overview of Kampo medicine: Toki-Shakuyaku-San (TJ-p23)," Phytotherapy Research, vol. 7, no. 6, pp. 391-394, 1993.

[7] S. Sakamoto, N. Hagino, and K. Toriizuka, "Effect of TokiShakuyaku-San (TJ-23) on the activity of choline acetyltransferase in the brain of menopausal rats," Phytotherapy Research, vol. 8, no. 4, pp. 208-211, 1994.
[8] Y. Kitabayashi, K. Shibata, T. Nakamae, J. Narumoto, and K. Fukui, "Effect of traditional Japanese herbal medicine tokishakuyaku-san for mild cognitive impairment: SPECT study," Psychiatry and Clinical Neurosciences, vol. 61, no. 4, pp. 447448, 2007.

[9] Q. Yang, H. Goto, H. Hikiami et al., "Effect of Tokishakuyaku-san on microcirculation of bulbar conjunctiva and hemorheological factors in patients with asymptomatic cerebral infarction," Journal of Traditional Medicines, vol. 21, pp. 170-173, 2004.

[10] M. Liu, T. Tsuji, K. Tsujiuchi, and N. Chino, "Comorbidities in stroke patients as assessed with a newly developed comorbidity scale," American Journal of Physical Medicine and Rehabilitation, vol. 78, no. 5, pp. 416-424, 1999.

[11] N. Chino, S. Sonoda, K. Domen, E. Saitoh, and A. Kimura, "Stroke impairment assessment set (SIAS)," in Functional Evaluation of Stroke Patients, N. Chino and J. L. Melvin, Eds., Springer, Tokyo, Japan, 1995.

[12] Center for Functional Assessment Research Uniform Data System for Medical Rehabilitation for USD Data Management Service: Guide for Use of the Uniform Data Set for Medical Rehabilitation Including the Functional Independence Measure (FIM), UB Foundation Activities, New York, NY, USA, 1994, ver 4 , Buffalo.

[13] R. Cote, R. N. Battista, C. Wolfson, J. Boucher, J. Adam, and V. Hachinski, "The Canadian neurological scale: validation and reliability assessment," Neurology, vol. 39, no. 5, pp. 638-643, 1989.

[14] T. Brott, H. P. Adams Jr., C. P. Olinger et al., "Measurements of acute cerebral infarction: a clinical examination scale," Stroke, vol. 20, no. 7, pp. 864-870, 1989.

[15] "Task Force on Stroke Impairment, Task Force on Stroke Disability, and Task Force on Stroke Handicap, Symposium recommendations for methodology in stroke outcome research," Stroke, vol. 21, supplement 2, pp. 68-73, 1990.

[16] K. Iwasaki, J.-C. Cyong, S. Kitada et al., "A traditional Chinese herbal medicine, banxia houpo tang, improves cough reflex of patients with aspiration pneumonia," Journal of the American Geriatrics Society, vol. 50, no. 10, pp. 1751-1752, 2002.

[17] T. Suzuki, S. Futami, Y. Igari et al., "A Chinese herbal medicine, choto-san, improves cognitive function and activities of daily living of patients with dementia: a double-blind, randomized, placebo-controlled study," Journal of the American Geriatrics Society, vol. 53, no. 12, pp. 2238-2240, 2005.

[18] K. Terasawa, Y. Shimada, T. Kita et al., "Choto-san in the treatment of vascular dementia: a double-blind, placebocontrolled study," Phytomedicine, vol. 4, no. 1, pp. 15-22, 1997.

[19] N. Kotani, T. Oyama, I. Sakai et al., "Analgesic effect of a herbal medicine for treatment of primary dysmenorrheal - a doubleblind study," The American Journal of Chinese Medicine, vol. 25, pp. 205-12, 1997.

[20] T. Tanaka, "A novel anti-dysmenorrhea therapy with cyclic administration of two Japanese herbal medicines," Clinical and Experimental Obstetrics and Gynecology, vol. 30, no. 2-3, pp. 95-98, 2003.

[21] B. Pan, Y. Kato, K. Sengoku, N. Takuma, N. Niizeki, and M. Ishikawa, "Treatment of climacteric symptoms with herbal formulas of traditional Chinese medicine," Gynecologic and Obstetric Investigation, vol. 57, no. 3, pp. 144-148, 2004.

[22] S. Usuki, T. Nakauchi, S. Higa, and K. Someya, "The improvement of luteal insufficiency in fecund women by Tokishakuyakusan treatment," American Journal of Chinese Medicine, vol. 30, no. 2-3, pp. 327-338, 2002. 
[23] S. Higaki, T. Toyomoto, and M. Morohashi, "Seijo-bofuto, Jumi-haidoku-to and Toki-shakuyaku-san suppress rashes and incidental symptoms in acne patients," Drugs under Experimental and Clinical Research, vol. 28, no. 5, pp. 193-196, 2002.

[24] K. Yamada, "Quality of life in patients treated with Kampo medicine: a complementary alternative to modern medicine," Journal of Alternative and Complementary Medicine, vol. 12, no. 8, pp. 799-803, 2006.

[25] K. Higashi, H. Rakugi, H. Yu, A. Moriguchi, T. Shintani, and T. Ogihara, "Effect of kihito extract granules on cognitive function in patients with Alzheimer's-type dementia," Geriatrics \& Gerontology International, vol. 7, pp. 245-251, 2007.

[26] K. Iwasaki, S. Kanbayashi, Y. Chimura, M. Taguchi, K. Inoue, S. Cho et al., "A randomized, double-blind, placebo-controlled trial of the Chinese herbal medicine "Ba Wei Di Huang Wan" in the treatment of dementia," Journal of the American Geriatrics Society, vol. 52, pp. 1518-1521, 2004.

[27] H. Shinno, Y. Inami, T. Inagaki, Y. Nakamura, and J. Horiguchi, "Effect of Yi-Gan San on psychiatric symptoms and sleep structure at patients with behavioral and psychological symptoms of dementia," Progress in NeuroPsychopharmacology and Biological Psychiatry, vol. 32, pp. 881-885, 2008.

[28] N. Egashira, K. Iwasaki, Y. Akiyoshi et al., "Protective effect of Toki-shakuyaku-san on amyloid beta25-35-induced neuronal damage in cultured rat cortical neurons," Phytotherapy Research, vol. 19, pp. 450-453, 2005.

[29] M. Ito, M. Ohbayashi, M. Furukawa, and S. Okoyama, "Neuroprotective effects of TJ-23 (Tokishakuyakusan) on adult rat motoneurons following peripheral facial nerve axotomy," Otolaryngology-Head and Neck Surgery, vol. 136, pp. 225230, 2007.

[30] A.-Y. Shen, T.-S. Wang, M.-H. Huang, C.-H. Liao, S.-J. Chen, and C.-C. Lin, "Antioxidant and antiplatelet effects of DangGui-Shao-Yao-San on human blood cells," American Journal of Chinese Medicine, vol. 33, no. 5, pp. 747-758, 2005.

[31] A. Kawamura, M. Iacovidou, A. Takaoka, C. E. Soll, and M. Blumenstein, "A polyacetylene compound from herbal medicine regulates genes associated with thrombosis in endothelial cells," Bioorganic and Medicinal Chemistry Letters, vol. 17, no. 24, pp. 6879-6882, 2007.

[32] M. Yamada and T. Ikeda, "How far is drug therapy possible for Alzheimer type dementia?" Asian Journal of Medicine, vol. 36, pp. 45-50, 1993.

[33] B. Ramírez-Ruiz, M.-J. Martí, E. Tolosa et al., "Cerebral atrophy in Parkinson's disease patients with visual hallucinations," European Journal of Neurology, vol. 14, no. 7, pp. 750-756, 2007.

[34] S. Yamaguchi, M. Matsubara, and S. Kobayashi, "Event-related brain potential changes after Choto-san administration in stroke patients with mild cognitive impairments," Psychopharmacology, vol. 171, pp. 241-249, 2004. 


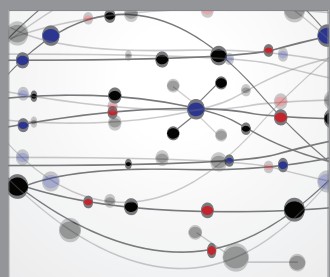

The Scientific World Journal
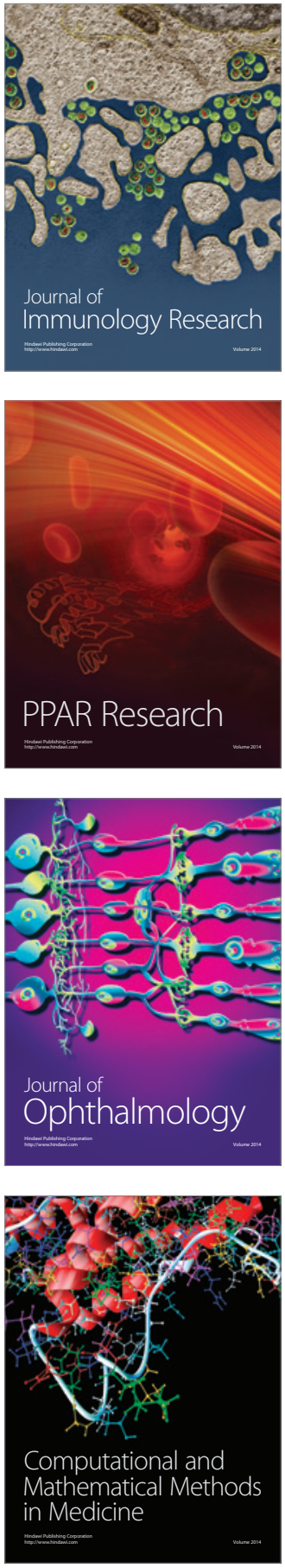

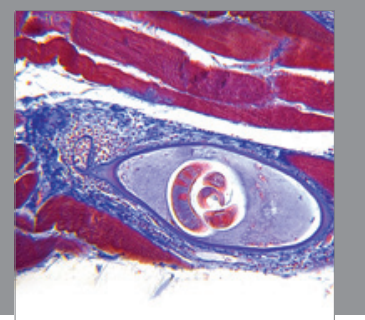

Gastroenterology

Research and Practice
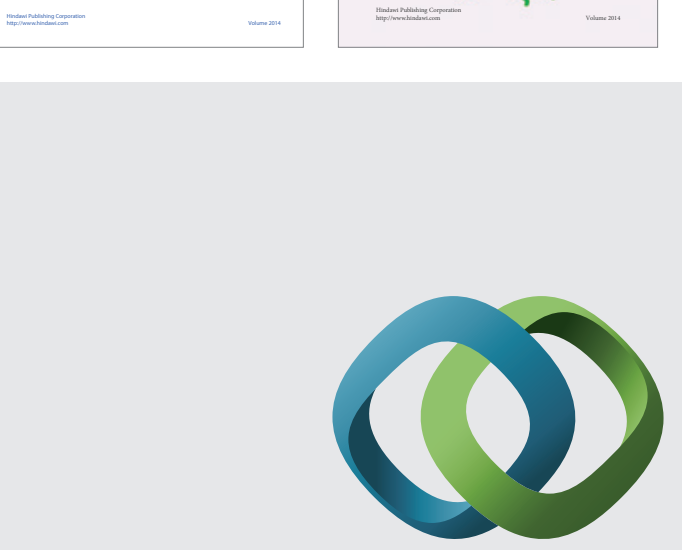

\section{Hindawi}

Submit your manuscripts at

http://www.hindawi.com
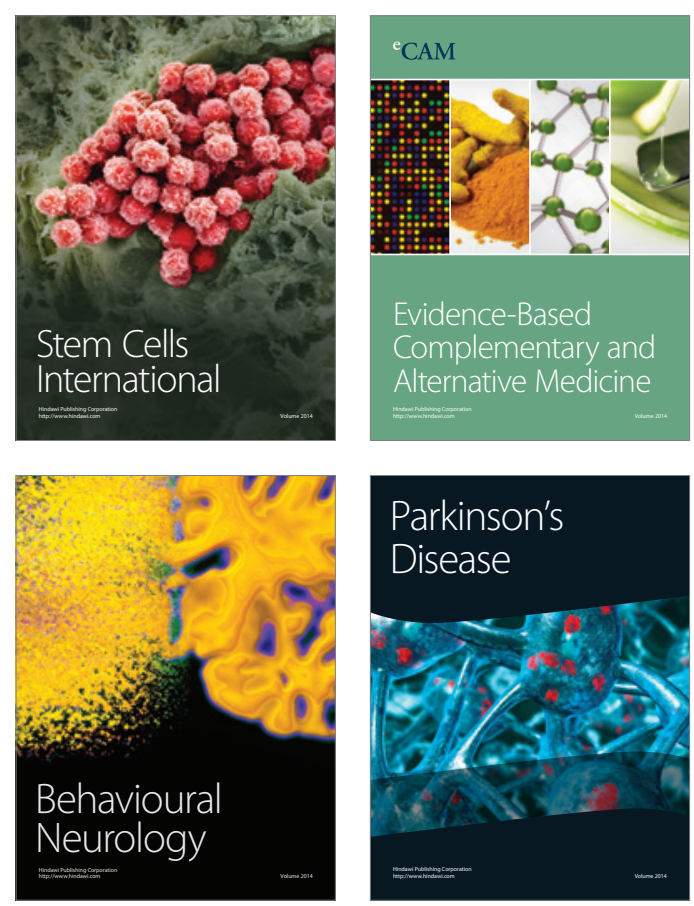

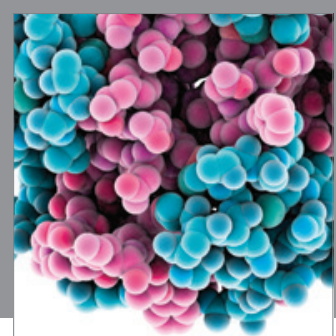

Journal of
Diabetes Research

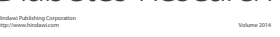

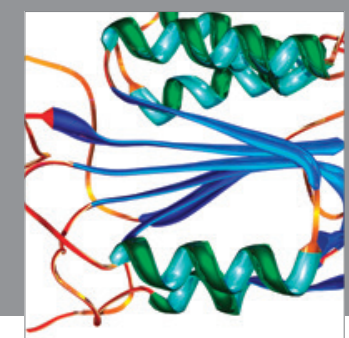

Disease Markers
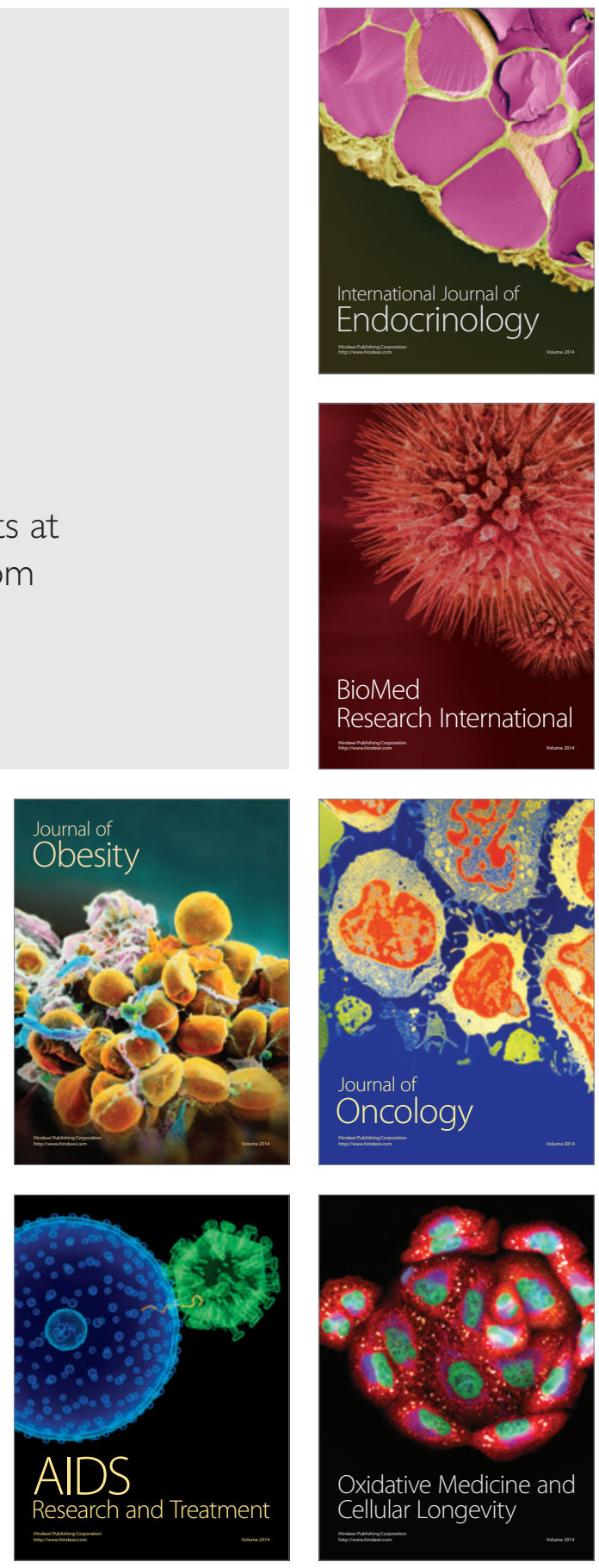